\title{
Dermatomiositis juvenil: manifestaciones clínicas y de laboratorio
}

\author{
Marta Miranda A.' ; Aurelio Carvallo V. ${ }^{1}$ \\ Juvenile dernatomyositis: clinical manifestations \\ and laboratory findings in 10 cases
}

Juvenile dermatomyositis (JDM) is a chronic, inflamatory degenerative entity with multisystemic involvement, particula:ly of sk in and striated muscle. This is a description of the clinical features, laboratory findings and treatment results in 10 childen with JDM wich were under control along a 14 years period (1974-1988) in a general hospital at metropolitan Santiago. Chile. Onset was insidious in B cases and acute in the remaining patients. All chitdren had muscle and $5 k$ in involvement, 90\% of them showed heilotrops erythema and 6 cases lamong the total 10) had one or more additiona! skin signs. Ninety percent of patients had risen serum activity of muscle associated enzimes, lactic dehydrogenase $(\mathrm{LDH}$ ) being the most constantly elevated: $7 / 7$ studied cases. All patients showed abnormal electromyographic and hystologic findings in muscle biopsy. Treatment was based upon rehabilitation, prednisone (average daily dose: $1.5 \mathrm{mg} \cdot \mathrm{kg}$ of body weight) associated or not with cytotoxic drugs, accordingly to each individual case. Clinical course was considered to be favourable in 9 of the 10 cases because ail of them showed no evidence of disease activity on last follow up control 26 to 93 months after initiai consultation, six had only slight restriction to wider range movements of affected joints and three showed no sequeiae. On'y one death occured in this serie, to a patient who rejected treatment,

(Key words: dermatomyositis, clincal signs, laboratory findings, treatment.)

La dermatomiositis juvenil (DMJ) es una enfermedad inflamatoria crónica, degenerativa, multisistemica, donde el compromiso de musculatura estriada y de piel son hechos relevantes.

1. Unidad de Reumatología, Hospital San Juan de Dios. Servicio y Departamento de Pediatría. Facultad de Medicina Occidente. Universidad de Chile.
De acuerdo a los criterios diagnôsticos descritos por Bohan y Peter ${ }^{1}$ la DMJ se caracteriza por eritema heliotrópico de la piel y tres de los siguientes criterios: debilidad muscular proximal sinétrica; elevación sérica de enzimas musculares; evidencia de vasculitis o inflamación crónica en la biopsia muscular y anormalidades electromiográficus sugerentes de miopatía inflama. toria. 
En los niños la afección tiene hechos que la distinguen de la enfermedad del adulto. En ellos la vasculitis es responsable de la mayoria de las manifestaciones clínicas, es rara su asociación a neoplasias y muestra alta incidencia de calcinosis distróficas en las etapas tardias ${ }^{2-7}$.

La etiologia de la DMJ es desconocida: sin embargo, ciertos hechos sugieren participacion de virus, en especial enterovirus, sobre todo del grupo Coxsackje $B^{8,9}$, que actuarian sobre un terreno susceptible ${ }^{10,11}$, el cual se expresaría con una respuesta inmune inapropiada, consti. tuida por prolucción de diversos tipos de anti. cuerpos $^{12,13}$, participación de los sistemas del complemento, de la coagulación ${ }^{14}$ y altera. ciones de la inmunidad celular ${ }^{15,16}$.

La frecuencia de DMJ en la población general se desconoce, pero en centros de referencia de reumatología infantil, incluido el nuestro, ocupa el tercer lugar entre las diversas afecciones del tejido conectivo.

En consjdetación a la escasez de referencias nacionales ${ }^{17-20}$ se hizo este análisis de los aspectos clinicos, de laboratorio, terapia y evolución con el propósito de describir el comportamiento e identificar elementos de juicio que permitan plantear precozmente el diagnóstico y el manejo de esta enfermedad.

\section{Pacientes y Método}

Se analizaron los casos de pacientes controlados en el Departamento de Reumatologia del Hospital San Juan de Dios de enero de 1974 a diciembre de 1988. Diez cumplieron con los criterios de diagnóstico señaladus por Bohan $y$ col. ${ }^{\prime}$ y constituyen la base de este trabaju.

Se revisaron los diagnósticos iniciales. La forma de comienzo tue caljficada según el tiempo que tardó la instalación de las manifestaciones clínjcas en: aguda (menor de cuatro semanas), subaguda (1 a 12 meses), insidiosa o cróniça (más de 12 meses). Se registraron, ađemás, el tiempo de evolución al consultar; las manifestaciones clínjcas aurante la evolución de la enfermedad; hemograma; velocidad de sedimentación eritiocitaria; exámenes serológicos (electroforesis de proteínas, factor reumatojdeo con técrica de látex, anticuerpos antinucleares con técnica de inmunofluorescencia indirecta, células de lupus); estudio enzimático (creatinfosfoquinasa, transaminasa glutámico oxalacética y pirúvica, deshidrogenasa láctica). En 9 pacientes se determinaron 3 a 4 clases de enzimas; en un enfermo se estudjó sólo una (transaminasa glutámico oxalacética); en todos, excepto en un niño, las enzimas se midieron antes del tratamiento esteroidal, siempre precediendo al estudio electromiográfico e histológico $\mathrm{y}$, luego, periódicamente durante la evolución. El đaxto muscular también se evaluó en forma indirecta a tra- vés de creatinuria de 24 horas. En todos los casos se completó el estudio con electromjografía y biopsia muscular. La respuesta al tratamiento y la evolución, incluyendo su estado de salud actual, fueron evaluados mediante criterios clínicos (compromiso dérmico, muscu. lar, articular $y$ visceral) y de laboratorio (enzimas musculares $y$ creatinuria de 24 horas).

\section{Resultados}

Nueve pacientes eran mujeres. La edad en que se inició la enfermedad fue entre 4,6 y 12 años $(\bar{x}: 8,4$ a) $y$ el periodo de seguimiento fue de 26 a 93 meses $(\bar{x}: 50,5 \mathrm{~m})$. El djagnostico inicial fue incorrecto en todos los casos, siendo más frecuente la confusión con orra mesenquimopatia en 8 oportunjdades, seguido por dermitis alérgica en 4 ocasiones. Dos casos llegaron con e] diagnóstico de DMJ y habian recibido trata. miento esteroidal previo, pero en su inicio también se plantearon diagnósticos erróneos. El tiempo de evolución al consultar fluctuó entre menos de un mes ( 1 caso) y más de 12 meses (4 casos).

Las manifestaciones clinicas fueron de comienzo agudo en dos niños, subagudo en cuatro e insidioso, mayor de 12 meses, en los cuatro restantes. Los hechos clínicos más relevantes se resumen en la tabla 1 , donde se ve que todos

Tabla 1

Manifestacjones clínicas en 10 njfios con dermatomiosit is juvenil

\begin{tabular}{lc}
\hline Síntomas y signos & n pacientes \\
\hline Debilidad muscular & 10 \\
Lesiones cutáneas & 10 \\
- Eritema en heliotropo & 9 \\
- Maculopápulas & 6 \\
- Ulceras curáneas & 4 \\
- Calcinosis & 4 \\
Mialgia & 8 \\
Artralgja & 8 \\
Artritis & 7 \\
Astenia, adinamia & 6 \\
Fiebre & $\mathbf{4}$ \\
Esclerodactilia & 4 \\
Disfragia & 4 \\
Calsinosis & $\mathbf{4}$ \\
Prurito & $\mathbf{4}$ \\
Fenómeno de Raynaud & 3
\end{tabular}


presentaban evidencia de compromiso muscular y cutáneo. La debilidad muscular de la cintura pélvica precedió a la escapular; más de la mitad de los casos (8 pacientes) presentó compromiso de la musculatura flexora del cuello y el tronco, un caso cursó con disfonia, por compromiso de musculatura faringea posterior. En $90 \%$ de los pacientes las alteraciones cutáneas fueron tipicas (eritema en heliotropo). Seis casos presentaron, además, otros tipos, isespecíficos, de lesiones (máculas o maculopápulas eritematosas de distribución irregular). En $4(40 \%)$ pacientes ocurrieron vasculitis cutánea y calcinosis, esta úlitima en los niños que tenian más de 1 año de evolución. La vasculitis se expresó en la piel de los cuatro pacientes como úlceras cutáneas que afectaban preferentemente las manos, dos de ellos tuvieron, además, signos de vasculítis digestiva (crisis de dolor abdominal recurrente) $y$ los dos restantes intensa miopatia. En $60 \%$ de los casos hubo algún grado de compromiso general (caracterizado por astenia, adinamia o pérdida de peso), $40 \%$ cursaron con fiebre en algún momento de la evolución.

Otras manifestaciones que se presentacon con menor frecuencia fueron: compromiso pulmonar (disnea, tos. dolor torácico, con signos radiológicos de alteraciones intersticiales basales y pruebas de función respiratoria alteradas de predominjo restrictivo), hepatomegalia y esple. nomegalia en un caso; derrame seroso pleural y dolor abdominal recurtente en dos ocasiones; adenomegalias en 3 pacjentes $y$ alopecia en un caso.

En los hemogramas la serie roja era normal en todos los pacientes, excepto on uno, que cursó con anemia nomnocitica normocrónica; habia Jeucocitosis mayor que $11.000 \mathrm{x} \mathrm{m}^{3}$, sin desviación a izquierda en 2 casos y una enferma presentó trombocitosis persistente sobre 400.000 plaquetas $\mathrm{x} \mathrm{mm}^{3}$; la velocidad de sedimentación eritrocitaria estuvo elevada en 8 de los 10 casos, siendo sólo en dos de ellos mayor que $50 \mathrm{~mm} / \mathrm{h}$.

La búsqueda de anticuerpos antinucleares dio resultados positivos en 4 de 8 examenes efectuados (tres con patrón granuloso y uno homogéneo): la electroforesis de proteinas mostró en 5 de 6 pacientes hipoalbuminemia relativa y aumento global de las gammaglobulinas. El factor reumatoideo y las células de lupus fueron negativos en los 10 casos.

Las enzimas de origen muscular estuvieron elevadas en todos los casos, con la excepción de una niña que ya recibia tratamiento esteroidal. Como se observa en la tabla 2 , la deshidrogenasa láctica fue la enzima de mayor sensibilidad. La creatinuria de 24 horas estuvo elevada en $9 / 10$ casos con respecto a su peso y edad. El único paciente con valores normales fue el mismo casu en que no se demostró alza de las tasas enzimáticas. En todos hubo adecuada correla. ción entre actividad alterada de las enzimas y aumento de la creatinuria.

La electromiografía estaba alterada en todos los casos, destacando la evidencia de irritabilidad de membrana (fibrilaciones y ondas positivas) y potenciales de acción voluntaria disni. nuidos de amplitud y duración, de carácter po. lifásico.

En la tabla 3 se detailan los principales hechos histológicos de la biopsia muscular, que permitieron fundamentar el diagnóstico. Todos los pacientes tuvieron evidencia de polimiositis. En 7 de los 10 casos se analizaron las manifestaciones histopatológicas cutáneas, encontrándose en 5 elementos de vasculitis y en 4 aumento

Tabla 2

Resultados de Jas mediciones de actividad enzinática en el suero de 10 pacientes con dermatomiositis juvenil al ingreso

\begin{tabular}{|c|c|c|}
\hline Enzimas & $\begin{array}{l}\text { Anormales; } \\
\text { estudiados }\end{array}$ & Porcentaje \\
\hline Creatinfosfoqujnasa & 519 & 56 \\
\hline Deshidrogenasa láctica & $7 / 7$ & 100 \\
\hline $\begin{array}{l}\text { Transaminasa glutámico } \\
\text { oxalacética }\end{array}$ & $7 / 9$ & 78 \\
\hline $\begin{array}{l}\text { Transaminasa glutámico } \\
\text { pirúvica }\end{array}$ & $6 / 8$ & 75 \\
\hline
\end{tabular}

Tabla 3

Hallazgos histopatológicos en biopsias de músculo estriado en 10 pacientes con dermatomiositis juvenil

\begin{tabular}{ll}
\hline Tipo de alteración & $\mathbf{n}$ \\
\hline Degeneración & 8 \\
Regeneración & 7 \\
Fibrosís & 6 \\
Inflamación & 4 \\
Variedad de diámetro & 3 \\
Fagocitosis & $\mathbf{1}$
\end{tabular}


del tejido conectivo, con fibras colágenas que sobrepasaban el nivel de las glándulas sudoríparas, sugiriendo esclerodermia.

El tratamiento incluyó teposo (durante el periodo de mayor actividad), rehabilitación pre$\operatorname{coz}$ y corticosteruides. En 3 casos se emplearon, además, inmunosupresores (azatioprina), ya sea para control de la actividad de la enferme. dad (un caso) o para suprimit los esteroides, debido a sus complicaciones ( 2 casos). La dosis de prednisona fluctuó entre 0,33 y $2 \mathrm{mg} \cdot \mathrm{kg}$ · día (promedio $1,5 \mathrm{mg} \cdot \mathrm{kg} \cdot \mathrm{dia}$ ). En 5 casos, por mal resultado de la terapia, se cambió por otro esteroide (metilprednisona o triamcinolona), obteniéndose respuesta adecuada en 4 de ellos, pero en el quinto fue necesario usar inmunosupreso. res. La reducción de la dosis se efectuó basándose en la respuesta clínica y el control enzimático. El tiempo promedio de tratamiento fue de 30 meses.

Entre las complicaciones del tratamiento se observaron infecciones repetidas de vías respiratorias bajas en dos pacientes; tuberculosis renal en un caso; infección urinaria reiterada en otro y herpes zóster en dos niffos. Secundariamente al uso crónico de esteroides se produjo en todos los pacientes sindrome de Cushing iatrogénico, en 4 ocurrieron fracturas patológicas por osteoporosis (tres en cuerpos verterales y una en fémur), dos niños tuvieron glaucoma y uno catarata subcapsular posterior.

$\mathrm{La}$ actividad de la enfermedad tuvo una duración que varió entre 24 y 78 meses, $\bar{x}$ : 48 neses. No se observó relación entre el inicio agudo, subagudo a crónico y la evolución posterior; sin embargo, aquellos pacientes con compromiso muscular severo al ingresar (postrados en cama, 4 casos) tuvieron un curso más prolongado (rango 3 a 6,6 años, $\bar{x}: 5$ años). De los 10 pacientes, uno abandonó el tratamiento y falleció; los 9 restantes están vivos, todos con su enfermedad inactiva, sólo 2 permanecen aún en tratamiento con esteroides en bajas dosis, 3 están sin secuelas y 6 presentan retracciones musculares que limitan los rangos extremos de movilidad articular, pero no les impiden realizar una vida normal.

\section{Discusión}

La dermatomiositis juvenil (nombre utilizado para la enfermedad que comienza antes de los
16 años) es una entidad poco frecuente entre los niños, al igual que el resto de las afecciones reumatologicas, cuya frecuencia se estima en $2 \%$ de la morbilidad pediátrica ${ }^{21-23}$. Si consideramos sólo a las afecciones del tejido conectivo. la DMJ corresponde a 4 ó $6 \%$ de las mismas, precediéndola en frecuencia la artritis reumatoidea juvenil $(75$ a $83 \%$ ) y el lupus eritematoso sistémico $(8,5$ a $10 \%)$. En nuestro centro, donde se atienden pacientes adultos y pediátricos, representa $27 \%$ de las polidermatomiositis ${ }^{24}$. En otras experiencias las proporciones difieran. asi Bohan ${ }^{1}$, en un centro reumatológico gene. ral, encontró sólo 7\%.

Con respecto a la edad de comienzo, nuestros datos concuerdan con otros que describen la mayor frecuencia entre $\operatorname{los} 4$ y 10 años ${ }^{2}$; sin embargo, existen casos descritos en lactantes $^{4}$. El franco predominio del sexo femenino encontrado en esta serie excede con mucho la relación $2: 1$ descrita por la mayoría de las publicaciones.

Si bien las manifestaciones clinicas relevantes están relacionadas con el compromiso muscular y cutáneo, el espectro es tan amplio y variado que explica el diagnóstico habjtualmente erróneo y tardío. Tanto el compromiso muscular como démico no difieren de lo ya descrito; destacamos las lesiones de vasculitis de piel, de características severas, asociadas en algunos con manjfestaciones sugerentes de vasculitis de nucosa gastrointestinal (crisis de folor abdominal re. currente), o miopatía intensa. En otra serie de 29 casos, 34\% tenia lesiones vasculares de piel y mucosas, cuya histología iba desde vasculitis linfocíticas no necróticas hasta lesiones endo. vasculares responsables de oclusión temporal o permanente de arterias pequefias y capilares, encontrándose correlación entre estas alteraciones y los hallazgos en otros órganos afectados?

La calcinosis es otro elemento característico de la DMJ, descrito en 40 a $75 \%$ de los casos. Suele hacerse evidente después de un tiempo de evolución de la enfermedad; sin embargo, Pachman señaló que en 2 de sus 9 pacientes se detectó al inicio de la afección y antes del tratamiento ${ }^{6}$, lo que descartaría el papel de los corticoides como factor desencadenante, rol que en alguna oportunidad se les atribuyó. Se ha descrito últimamente su relación con proteínas dependientes de vitamina $\mathrm{K}$ (proteinas que contienen el grupo gammacarboxilico de la 
glutamina), cuya excreción urinarja está aumentada en pacientes con polimiositis ${ }^{26}$.

A diferencia de la enfermedad del adulto, la DMJ excepcionalmente se ha descrito asociada a neoplasia ${ }^{5}$; esto también se consignó en nuestra serie.

Los exámenes complementarios orientados a demostrar daño muscular tuvieron una buena correlación con la clínica, destacando la alta sensibilidad de la enzima deshidrogenasa láctica. La normalización đe los valores de creatinuria en 24 horas fue más lenta que la de las enzimas. Pudimos confirmar la alta sensibilidad de la electromiografía señalada por otros autores ${ }^{1}$ y destacar la correlación que existe entre la fase aguda de la enfermedad y las manifesta. ciones de irritabilidad de membrana, traducida por potenciales de inserción aumentados y fíbrilaciones. Si bien la biopsia muscular estaba al. terada en todos los pacientes, nos llamó la aten. ción la ausencia de vasculitis, que se describe como hecho relevante de la DMJ, pues Crowe ${ }^{\text {? }}$ seriala que existía en $48 \%$ de su serie en niños. a diferencia lo que ocurre en adultos, en quienes Bohan ${ }^{1}$ encontró sólo cuatro casos entre 153 pacientes. No tenemos una explicación satisfactoria para este hecho. Sin embargo, encontramos vasculitis en la biopsia de piel en 7 casos, agregándose en 4 de los mismos, aumento del tejido colágeno similar al obseryado en la esclerodermia. Se ha planteado la hipótesis que dichos cambios sean secundarios al estímulo que representa la hipoxia crónica para la síntesis de colágeno ${ }^{27}$.

En lo referente al tratamiento, la prednisona a altas dosis constituye la medida de elección y su uso preco 2 puede cambiar el curso de la enfermedad; se ha postulado que en aquellos pacientes que evolucionan con hepatomegalia como expresión de su compromiso sistémico, la metilprednisolona tiene mejor resultado, probablemente por inadecuada hidroxilación hepática de la prednisona ${ }^{28}$. El uso de inmunosupresores, especialmente metotrexato y azatioprina, se reserva para casos severos que no responden a los esteroides ${ }^{29-31}$ hechos confirmados en nuestros pacientes.

E1 pronóstico favorable en $90 \%$ de los pacientes está de acuerdo con rango de 78 a $88 \%$ de otras series ${ }^{2,6}$, to que implica buenas perspectivas para estos pacientes, en especial cuando el diagnóstico es precoz, y el tratamiento ade. cuado,

\section{Resumen}

La dermatomiositis juvenil (DMJ) es una eת. fermedad inflamatoria cronica, degenerativa, multisistémica, donde el compromiso muscular estriado y de piel es relevante. El objetivo de nuestro estudio fue reevaluar sus manifestaciones clínicas, de laboratorio y su evolución en la ninez. Se analizaron 10 pacientes que cumplían los criterios de diagnóstico conmúnmente aceptados y que habían sido seguidos durante un período de 14 años (1974 a 1988). Predominaron en ellos las formas de comienzo insidioso (8 niños) sobre el agudo ( 2 niños). Todos tuvieron manifestaciones musculares y cutáneas, 90\% presentó eritema heliotropo y en 6 casos hubo. además, otro tipo de alteraciones dérmicas. De los exámenes complementatios, 90\% de los casos mostró alza de la actividad sérica de enzimas relacionadas con el músculo estriado, siendo la más sensible la deshidrogenasa láctica $y$ todos tenian alteraciones electromiograficas e histológicas. El tratamiento consistio en rehabilitación, corticosteroides (x 1,5 $\mathrm{mg} \cdot \mathrm{kg} \cdot \mathrm{dia}$ ) asociándose citotóxicos de acuerdo al caso. Con estas medidas se obtuvo buena respuesta en $90 \%$ de los pacientes. El único paciente que falleció fue también el único que rechazó el tratamiento.

(Palabras clave: dermatomiositis, manifestaciones clínicas, laboratorio, tratamiento.)

\section{Referencias}

1. Bohan, A.; Peters, J.B.; Bowman, R.L. and Pearson. C.M.: A computer assited analysis of 153 patients with polymyositis and dermatomyositis. Medicine 1977;56:255-286.

2. Sulfian, D.B.: Cassidy, J.T and Petry, R.E.: Dermatomyositis in the pediatric patient. Arthritis Rheum 1977, 20 (Suppl) 327-331.

3. Cook, C.D.; Rosen, F.S ond Banker, B.Q.: Dermatomyositis and focal scleroderma. Pediatr Clin North Am 1963; 10: 979.1016.

4. Hanson, $V_{\text {: }}$ Kornreich, $H,:$ Systemic rheumatic disorders. Collagen disease in childhood: Lupus erythematosus, anaphylactoid purpura, dermatomyositis and scleroderna. Bull Rheum Dis 1967; 17: $441-446$.

5. Tuffanelli, $D$. and Lo Perriere, $R$.: Connective Tissue Disease. Pediatr Clin North Am 1971; 18: $925-950$.

6. Pachmon, L.M.: Cooke, N.: Juvenile dermatomyositis: A clinical and inmunolugic study. J Pediatr 1980; 96 : 226-234. 
7. Crow'e, N.E;; Bore, K.E.; Levinson, J.E. and Hitton, $P, K$.: Clinical and pathogenic implications of histoplathology in childhood polydermatomyositis. Arthritis Rheum 1982; $25: 126.139$.

8. Tang, T.T.: Sedmak, $G . V_{. ;}$Siegesmund, $K . A_{.}$: Chronic myopathy associated with coxsackie virus type Ag: A combined electron microscopic and viral isolation study. $\mathrm{N}$ Engl $\mathrm{J}$ Med 1975; 292: 608-611.

9. Christensen, M.L.; Pachman, L.M.; Schneiderman, R.; Patel, D.C. and firiedman, J.M.: Prevalence of coxsackies $B$ virus antibodies in patients with juvenile dermatomyositis, Arthritis Rheum 1986; 29: $1365-1370$.

10. Cannon, R.A.; Jonasson, O. and Pachman, L.M.: HLA phenotypes in childhood dermatomyositis. Arthrit is Rheum 1977; 20 (Suppl): 499.

11. Pachman, L.M.: Marvjowski, M.C.: Friedman, J.M. er al.: Immunogenetic studies of juvenile dermatomyositis: Increazed ocurrence of HLA B8, DRW3 and their relation to PM-1 and other autoantibodies. Arthritis Rheum, 1980; 23: 728.

12. Wolfe, J.A.: Adelstein, E. and Sharp. G.C.: Antinuclear antibody with destruct specificity polymyositis. J Clin Invest $1977 ; 59: 176-178$.

13. Nishikai, M.: Reichlin, M.: Heterogeneity of precipitating in polymyositis and Dermatomyositis: Characterization of the $10-1$ antibody system. Arthritis Rheum 1980; 23: 88]-888.

14. Pachman, L.M.: Scott, J.P.; Arroyale. C.M. et al.: Juvenile dermatomyositis: Activation of coagulation and complement system: Clin Res 1983; 31: 796 A.

15. Johnson, R.L.: Fink, C.W. and Ziff. M.: Lymphotoxin formation by Lymphocyte and muscle in polymyositis. J Clin Invest 1972; 51 : 2435-2438.

16. Miller, M.L.; L.antne?, R.: Pachman, L.M.: Natural and antibody dependent cellular cytotoxicity in children with systemic lupus erythematosus and juvenile dermatomyositis. J Rheumatol $1983 ; 10 ; 640-642$.

17. Olea, R.: Dermatomyosits: Arch Hosp Roberto del Rio, 1935 S: 78.92

18. Valdivieso, $R$. Y' Telesnik. I.: Dermatomiositis. Rev Med Chile 1941: 69: 44.

19. Jalil, J.: Armas-Cruz, R.: Ossandón, M. v Uhilla, V.: Dermatomiositis. Rev Med Chile: 1958; 86: $1-17$.
20. Palma, S.: Reyes, H.: Guzman, $L$ y Cartier, l... Dermatomiositis y Toxoplasmosis. Rev Med Chile 1982;111: 164-167.

21. Koempffer, A.; Medina, E.: Morbilidad y atención médica infantil en el (rian Santiago. Rev Chi) Pediatr 1980; 51 : 355-369.

22. Maddaleno, M, Florenzano, R.: Alvo, D. et al.: Estructura de morbilidad de adolescentes consultantes en el sector Oriente de Santiago. Rev Chil Pediatr 1987; 58: 164-168.

23. Jacobs, IC En: "Pediatric Rheumatology for the Practitioner. New York, Ed, Springer-Verlag 1982, Prefacio.

24. Carlollo, A.; Grisanti, M.; Aris, H.: Rojas, C. v Jolil, J.: Polimiositis: Análisis clínicu (presentado en las XIJI Jornadas Chilenas de Reumatología, lquique, diciembre 1978).

25. Cassidy, J.T.: Defïnition and classifjcation of Rheumatic Disease in children. En: Casidy, J.T.: Textbook of Pediatric Rhermatology. New York. John Willey \& Sons 1982, 1-13.

26. Lian, J.B.i Pachman, ls, M+i Ciundberg, C.M. Parridge, R.F.H. and Maryon'sky', M.C.: Gamma Carboxyglutamate excretion and calcinosis in juvenile dermatomyositis Arthritis Kheum 1983; 25: 1094-1100.

27. Bowyer, S.L.: Clark. R.A.F.: Ragsctale, C.G.. Hollister, J.R, and Sullivan, D.B.- Juvenjle dermatomyositis: Histolugical tindings and pathogenetic for the associated skin changes. $J$ Rheumatol $198,13: 753-759$.

28. Hoynes, R.C. It. and Murad, F.: Adrenocorticotropic hormone: Adrenocortical steroids and their synthetic analogs; inhibitors of adrenocortical steroid biosynthesis. En: The Pharmacologic Basic of therapeutics, 7th ed. Ed. Ag. Gilman, L.S. Goodman and A, Gilnuan. New Yotk. Macmillan Publishing Co, 1985.

29. Jacobs. J.C.: Metlrotrexate and azathiuprine treatment of childhood dernatomyositis. Pedjatrics $1977 ; 59: 212 \cdot 218$.

30. Laxer, R.M: Stein, L.D. and Pertl, R.E.: Intravenous pulse methylprednisolone treatment of juverile dermatomyositis, Arthritis and Rheum 1987; 30: 328-334.

31. Yoshioka, M.i Okuno, T, and Mikawa, H.: Prognosis and treatment of polymyositis with particular reference to steroid resistant patients. Arch Dis Child $1985 ; 60: 236-244$ 